\title{
The Discovery of Serotonin and its Role in Neuroscience
}

\author{
Patricia Mack Whitaker-Azmitia, Ph.D.
}

When reading the various stories of how serotonin was discovered, it immediately becomes apparent how fortuitous it was that serotonin was discovered at all. It started out as an annoying artifact that had to be gotten rid of before the real work of finding the cause of hypertension could be gotten to. It ended as one of the most important discoveries in neuroscience. Indeed, in many ways, serotonin gave birth to the field of neuroscience.

How did this good fortune arise? Clearly, the discovery was solely due to the uniqueness and genius of each of the researchers involved. Thus, to tell the study of how serotonin was discovered, it is best to describe the individual scientists and their goals, and how they came together to make their great discovery.

\section{VITTORIO ERSPAMER}

The story properly begins in the early 1930's with Vittorio Erspamer working in the Institute of Comparative Anatomy and Physiology, University of Pavia in Italy. Dr. Erspamer had originally planned to be a lawyer, but became interested in pharmacology and physiology. In particular, he was interested in the field of pharmacognosy-the study of drugs from natural sources. Even today, with all of the interest in natural herbal remedies, Dr. Erspamer remains unique in his devotion to the extraction of pharmacologically active substances from animals.

From the Department of Psychology, SUNY at Stony Brook, Stony Brook, NY.

Address correspondence to: Dr. P.M. Whitaker-Azmitia, Department of Psychology, SUNY at Stony Brook, Stony Brook, NY 11794.

Received December 17, 1998; accepted March 2, 1999.
In the 1930's, Dr. Erspamer was interested in the smooth muscle constricting or contracting properties of various amine substances found in the skins and intestinal tracts of a variety of species, including rabbits, mollusks, and frogs. One substance which interested him was found in enterochromaffin cells of the gut. An acetone isolate of the cells caused smooth muscle contraction, especially rat uterus. He deduced that the substance wasn't epinephrine and that on the basis of color tests, it was likely an indole. He named the substance enteramine (Erspamer and Vialli 1937) and continued studies on smooth muscle for several more years (Erspamer 1946). He also studied the mollusc heart (Erspamer and Ghiretti 1951) and the occurrence of enteramine in the salivary glands of the octopus (Erspamer 1948; Erspamer and Boretti 1951). Enteramine had a relatively extensive place in the scientific literature until 1952, when it was established as the same substance which Irvine Page, Maurice Rapport, and Arda Green had been working on since 1948, serotonin (Erspamer and Asero 1952). However, it was the work of Dr. Erspamer on enteramine which would later bring another key researcher into the story-Betty Twarog.

Since his discovery of enteramine, Dr. Erspamer has gone on to identify and characterize many other biologically important molecules, including bombesin, several opiates and a variety of neuropeptides which also can be isolated from amphibians. The expertise with which Dr. Erspamer has remained at the forefront of scientific research for over 60 years is amazing. Even Irvine Page (who could sometimes be a harsh critic) stated that "Erspamer must be recognized as one of the most productive and gifted investigators in the field." Dr. Erspamer is a member of the National Academy of Sciences. 


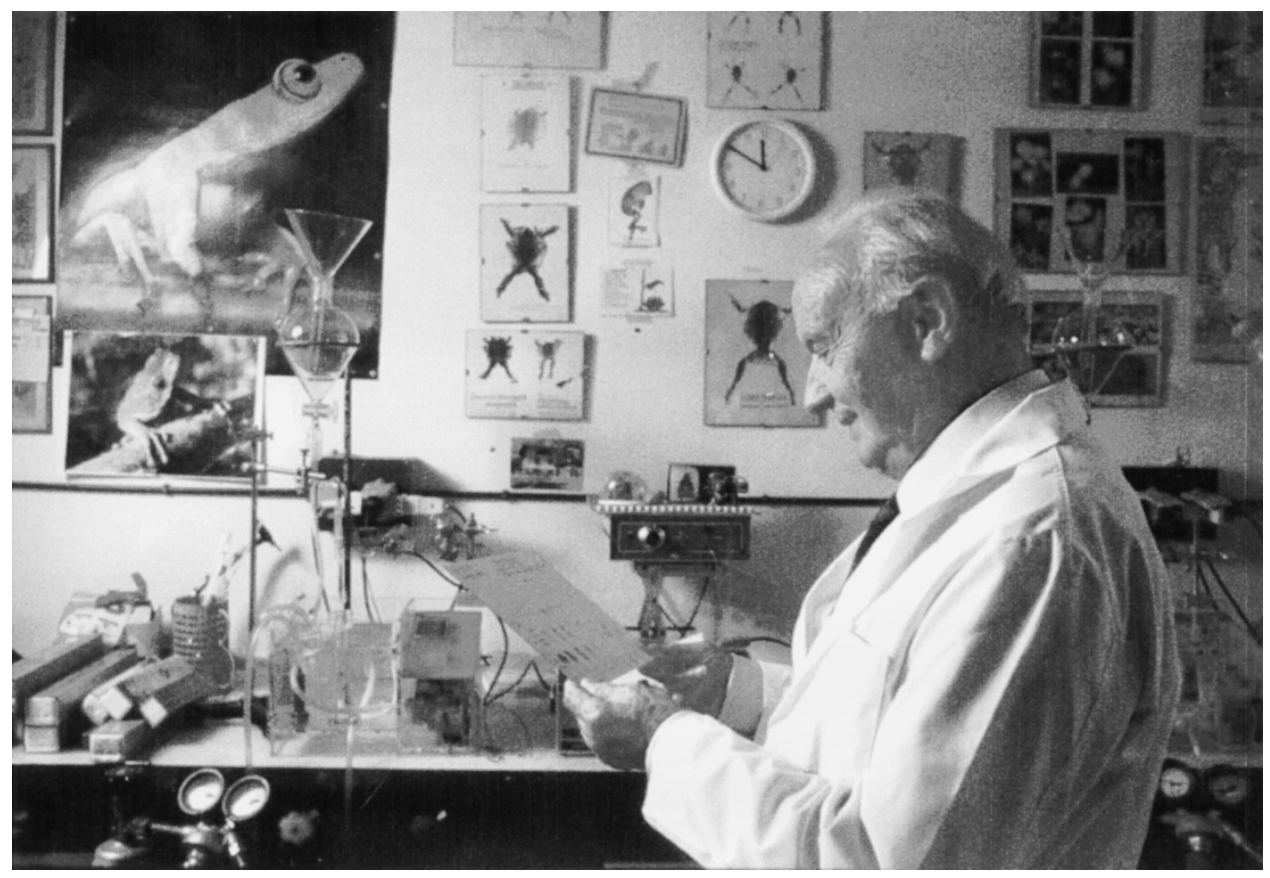

Figure 1. Vittorio Erspamer at work in his laboratory at the University of Rome.

In spite of Dr. Erspamer's other accomplishments, serotonin remains his favorite. In September of 1998, he wrote a letter of greeting to the participants of this symposium:

At the age of over 89 years, I remember with nostalgia the work of Vialli and myself in which the unknown secretory product of enterochromaffin cells was first extracted from the rabbit gastric mucosa and named enteramine... Through the extraordinary research by Rapport and co-workers on serotonin, this substance could be identified, exactly fifty years ago, as 5-hydroxytryptamine. Since then, an enormous amount of work has been done on the amine, especially after the discovery of its fundamental role in neurotransmission. Now, for more than twenty years, my research is focussed on neuropeptides from amphibian skin and invertebrate tissue, but I always consider enteramine as my firstborn daughter.

Why did the substance become known as serotonin rather than its first name, enteramine? The most likely explanation is that it was first synthesized and made available for research by the American drug company, Upjohn Pharmaceutical, who chose the name "serotonin."

Dr. Erspamer still works in his lab at the University La Sapienza in Rome (Figure 1). His wife, Giuliana, who is also a scientist, often works with him.

\section{IRVINE PAGE}

Irvine Page received his B.A. and M.D. from Cornell University and was interested in the etiology and treat- ment of essential hypertension. He began studying this problem at Eli Lilly, but in 1945 left to become the Director of Research at the Cleveland Clinic (Figure 2).

At that time, the Cleveland Clinic specialized in questions of hypertension and arteriosclerosis and the Research Division was also largely devoted to these issues. The research budget for the entire division was $\$ 160,000 /$ year. These funds were generated by the Clinic, the founders of which strongly believed in the importance of clinical and basic research to excellent medical care. The project on isolating vasoconstricting substances headed by Dr. Page, however, appears to have been funded primarily by a small grant of $\$ 12,500$ from the Life Insurance Medical Research Fund.

Principally, Dr. Page felt that hypertension could best be explained by the presence of endogenous constricting factors in blood. However, whenever blood was studied for such a factor, another substance was produced as soon as the blood coagulated. Dr. Page realized that this serum substance would have to be removed from the blood preparations before any progress could be made on the hypertension-producing factor. For this, Dr. Page had the foresight to recruit a highly skilled organic chemist, Maurice Rapport, and an equally skilled and distinguished biochemist, Arda Green. Page, Rapport, and Green succeeded in isolating and characterizing the serum substance and named it serotonin. These studies are summarized below, under the researcher who was principally responsible, Maurice Rapport.

After this time, Dr. Page turned back to the study of vasoactive substances, eventually being instrumental in the discovery of angiotensin. From his subsequent writ- 
A.

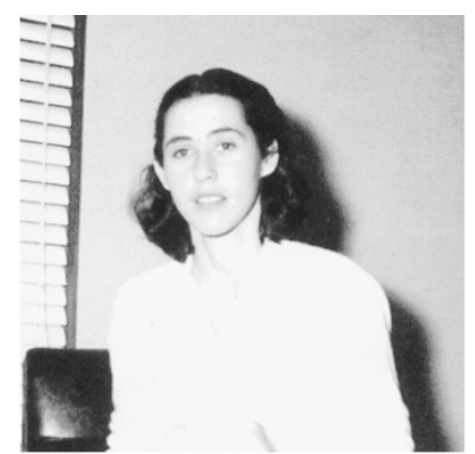

B.

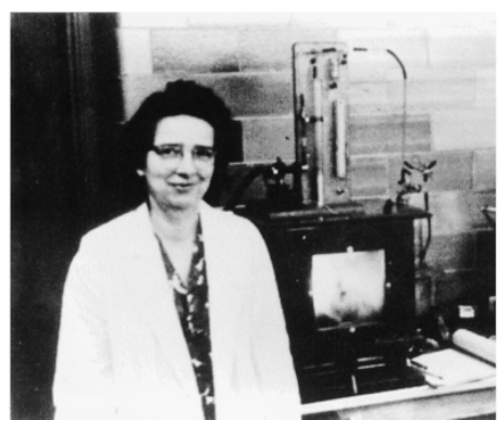

C.

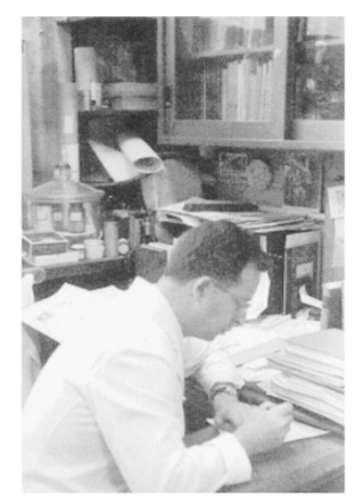

D.

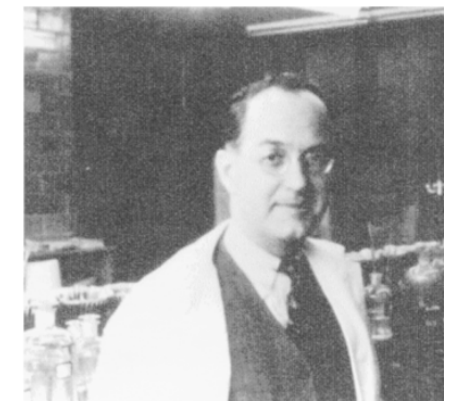

Figure 2. Early serotonin researchers. (A) Betty Twarog, 1952 (private collection); (B) Arda Green, 1947 (Cleveland Clinic Archives); (C) Maurice Rapport, 1948 (private collection); (D) Irvine Page, 1947 (Cleveland Clinic Archives). ing on the discovery of serotonin, it is clear that Page had mixed feelings. On the one hand he had participated in one of the most significant physiological discoveries of the century which brought him a considerable degree of fame. On the other, the discovery had not greatly advanced his own scientific interests in hypertension. In his book Serotonin, published in Page (1968) made a number of interesting and amusing statements which reflect these feelings:

"Thus, the past 20 years have been extraordinarily productive, resulting in thousands of papers (on serotonin), and even a great deal of valuable information." "I noted that serotonin had replaced epinephrine as a sure road to tenure for the pharmacologist."

"The great variety of suggested roles (for serotonin) can be said to be a tribute to man's ingenuity and his unquestionable willingness to write papers."

"Clearly, this field has fallen heir to the current disease of science-too many journals, too many meetings and too little worth talking about."

Irvine Page died in 1991 at the age of 90.

\section{ARDA ALDEN GREEN}

Arda A. Green, M.D. was already a highly accomplished biochemist when she was recruited by Irvine Page to work at the Cleveland Clinic in July of 1945. She had trained with John Evans, at UCLA and then joined the husband and wife research team of Carl and Gerti
Cori at Washington University, working out the catalysis of glycogen. During this work, Dr. Green was the first to isolate phosphorylase-A. In recognition of her work, when the Cori's received the Nobel Prize in 1947, they gave a significant share of it to Dr. Green.

At the Cleveland Clinic, Dr. Green took the rabbit ear artery preparation of Dr. Page and refined it further, for the study of blood components which could lead to vasoconstriction. Working with the rabbit ear was not easy because, in addition to the daily problems of standardization, the prep had a seasonal rhythm in its response. Any substances isolated by Dr. Green herself or later Maurice Rapport would be tested on this preparation. Most days, she would use the rabbit prep to test for vasoconstricting substances in kidney and was eventually instrumental in the discovery of angiotensin. At the end of the day, if the ear was still functional, Maurice Rapport would be allowed to try his isolates. In addition to providing Dr. Rapport with the ear prep, Dr. Green taught him the basic principles of pharmacology and physiology with which an organic chemist would be unfamiliar.

Dr. Green left the Cleveland Clinic in 1953, and took a position in the Department of Biology at Johns Hopkins University working on bioluminescent systems. Here, she was the first to isolate luciferase from fireflies. This enzyme continues to be an important part of many biochemical measuring systems and is a commonly used reporter gene. Dr. Green died of cancer in 1954, only six years after the discovery of serotonin. She was 57. In her career, she had been instrumental in the dis- 
covery of four substances-phosphorylase A, angiotensin, luciferase, and serotonin.

\section{MAURICE RAPPORT}

Maurice Rapport completed his Ph.D. in organic chemistry at Cal Tech in 1945. He was hoping to return to his hometown of New York, but with no job available, he took a position with Irvine Page who had been actively pressing him to come to the Cleveland Clinic. On arriving at the Clinic in February 1946, Dr. Rapport was not clear on what his assignment would be, but soon realized anything to do with isolating vasoconstrictors from blood would do. Dr. Rapport decided on isolating and eliminating the interfering artifact produced when blood clotted. In order to test for such a substance, he would ask Arda Green to test it in the rabbit ear artery.

Dr. Rapport needed vast quantities of blood combined with considerable scientific ingenuity to complete the task. Early in the morning, he would go to the Cleveland slaughterhouse, and collect eight 15 liter buckets of blood. Back at the lab, the blood was poured into a garbage can lined with cheesecloth with a small hole at the bottom. As the blood coagulated, the resultant serum dripped through the cheesecloth and out the hole. After two days in the cold room, 15 liters of serum were collected. More than sixty runs to the slaughterhouse were necessary to generate enough starting material. Through a five-step procedure (ethanol precipitation of proteins, acetone precipitation of salts, chloroform extraction of inactive substances, butanol extraction of the active substance, and finally, dilituric acid precipitation), a very small amount of the vasoconstrictor substance was isolated. This original sample, pictured in Figure 3, can still be seen at the Cleveland Clinic.

The isolation was reported to the Research Committee of the Clinic on March 9, 1948, in response to which they voted to give Maurice Rapport "a nice dinner party" for his discovery. The minutes of that meeting indicate that "The substance will be named Serotonin." In June 1948, the first publication describing the partial purification appeared in The Journal of Biological Chemistry (Rapport et al. 1948a)

Dr. Rapport continued working on the isolation. With two additional steps, an acetone precipitation to remove other solutes and a methanol extraction, he achieved success "thin, rhomboid pale-yellow (crystals) ... (which) we would like provisionally to name serotonin, which indicates its source is serum and its activity is one of causing constriction." The crystals are shown in Figure 3. This work appeared in Science on September 24, 1948 (Rapport et al. 1948b). However, the chemical structure was still unknown.
A.

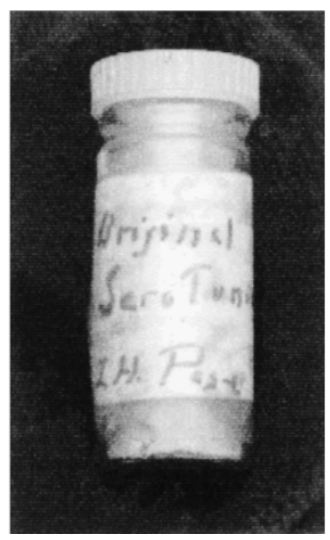

B.

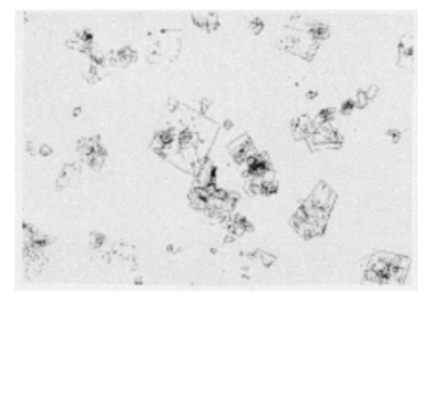

Figure 3. (A) Original vial of serotonin; (B) Original crystals of serotonin. Courtesy of the Cleveland Clinic Archives.

In the fall of 1948, Maurice Rapport left The Cleveland Clinic for a position at Columbia University, which he was offered providing he gave up his work on serotonin. However, in spite of his promise, he could not end the search for the structure. Eventually, Columbia University relented and even contributed $\$ 23$ to pay for the elemental analysis. In May 1949, the structure was finally determined to be 5-hydroxytryptamine (Rapport 1949). In many ways, this was the true discovery of serotonin, for now it could be truly identified and studied. Dr. Rapport collaborated with synthetic chemists at Upjohn Pharmaceutical and Abbott Laboratories until 1951, when synthetic serotonin was first made available for research. This was a major breakthrough for serotonin research-until that time the only way to study the physiological effects of serotonin was by isolating it from serum, much as Drs. Green and Rapport had done at the Cleveland Clinic.

After that time, Dr. Rapport worked at Sloan-Kettering on sphingolipids, but eventually he returned to the Psychiatric Institute at Columbia University. In 1969, he combined the departments of pharmacology, biochemistry and bacteriology into one of the country's first departments of Neuroscience. Dr. Rapport continued as chief until 1986, when the current head, Dr. John Mann, was appointed. Today, at almost 80, Dr. Rapport continues an active interest in neuroscience, attending many scientific meetings and conferences, to which he always adds his great enthusiasm and personal warmth.

Although serotonin had been isolated and synthesized by 1951, there still remained the question of its function. It had started out as an annoying contaminant in blood and many people, including Irvine Page, were disappointed that it probably had no use in treating disorders of vascular tone. The next great advance in the story of serotonin, was its discovery in brain. For that, Betty Twarog was key. 


\section{BETTY MACK TWAROG}

Betty Mack Twarog (a probable distant relative of mine!) was undertaking graduate studies in 1948 for an M.Sc. at Tufts College, when she heard a lecture by Kenneth Roeder on a phenomenon in mollusc muscles called catch. In the catch state, muscles remain contracted and resist stretch long after the period of excitation has passed. Catch is particularly convenient to investigate in the byssus retractor muscle of the edible mussel (Mytilus edulis). The byssal muscles orient the mussel, which is cemented to rocks, so that it resists detachment by the crashing waves and can collect food on the incoming tide. In 1949, Dr. Twarog started in the Ph.D. program at Harvard, with John Welsh as her mentor. For her research, she chose to study the smooth muscle membrane and to identify the neurotransmitters which regulated catch in Mytilus. John Welsh had long been examining possible neurotransmitters which affected heartrate in Mercenaria mercenaria (little neck clams or quahogs).

At that time, the neurotransmitter theory itself was still controversial, and only acetylcholine and norepinephrine had received any degree of recognition. By 1951, Dr. Twarog had identified the contracting neurotransmitter in Mytilus as acetylcholine, but could not identify the relaxing neurotransmitter. Norepinephrine was weakly active but it was evident that a new, as yet unidentified neurotransmitter was involved, which had chemical similarities to both epinephrine and the ergot alkaloids. When the papers on serotonin from the Cleveland Clinic came to Dr. Twarog's attention, she was interested because the structure fulfilled the structural requirements she had established for the Mytilus neurotransmitter.

However, serotonin was only a serum vasoconstrictor found in serum of mammals and there was as yet no reason to believe it would have any function as a neurotransmitter. That same year, Dr. Erspamer's papers showed that enteramine was found in octopus salivary glands and that this substance could excite mollusc heart. Both Betty Twarog and John Welsh were convinced that salivary secretions often contained toxins related to neurotransmitters. Thus, enteramine could be the unknown neurotransmitter which inhibited catch. Shortly after, when serotonin and enteramine were identified as the same substance, Drs. Twarog and Welsh requested serotonin from Abbott Laboratories and found that the unknown neurotransmitter was indeed serotonin. Although Dr. Twarog wrote the paper on these findings immediately, the paper was not actually published until two years later (Twarog 1954) because the Journal of Cellular and Comparative Physiology had not bothered to review a paper on an unknown neurotransmitter by an unknown author.
In the fall of 1952, for family reasons, Dr. Twarog was moving to the area of Kent State University. She drew a fifty-mile circle with a compass on a map, and determined that the Cleveland Clinic was close enough for her to do her studies and still raise her young son. Convinced that invertebrate neurotransmitters would also be neurotransmitters in vertebrates, she approached Irvine Page for support of these studies. Dr. Page immediately agreed, although he said that he did not believe serotonin would be found in brain. He gave Dr. Twarog a laboratory and technician. Working with the clam heart preparation, which she had refined to be extremely sensitive, Dr. Twarog found serotonin in mammalian brain. This brought serotonin into the field of neuroscience. To quote from Irvine Page's book:

When cerebral metabolism or "brain chemistry" was being established as a field worthy of study, serotonin played an extraordinary role. It will come as a surprise to younger readers that even as late as 1937 many scientists were dubious as to whether "neurochemistry" was indeed a discipline. If I had to select a single effect resulting from the discovery of serotonin, I would unhesitatingly suggest its influence in shaping investigators' ideas on cerebral activity.

This landmark paper was submitted in June of 1953, and appeared the following October (Twarog and Page 1953). Interestingly, that paper not only established serotonin along the pathway to recognition as a neurotransmitter, it also decreased the likelihood of its involvement in most cases of essential hypertension, as it contained measurements in urine of hypertensive patients which were comparable to controls.

Dr. Twarog left the Cleveland Clinic in 1954 and continued her work on invertebrate smooth muscle at Tufts, then Harvard, and later SUNY at Stony Brook. Today, she works at the Bigelow Laboratory for Ocean Sciences in Boothbay Harbor, Maine. She is still actively involved in research and is currently embarking on a research project with collaborators in Canada and America to elucidate the mechanisms whereby certain shellfish resist the potent neurotoxins of red tide phytoplankton.

Now, all that remained was to further expand the known role of serotonin in brain function.

\section{DILWORTH WAYNE WOOLLEY}

D.W. Woolley, Ph.D. was by any measure a gifted and unusual man. He believed that scientific progress could best be achieved by finding the unified meaning of apparently isolated and diverse facts. This is exactly what he did-by combining the work of Betty Twarog, showing serotonin existed in the brain, with Albert Hoffman's discovery of LSD, and his own work on LSD as 


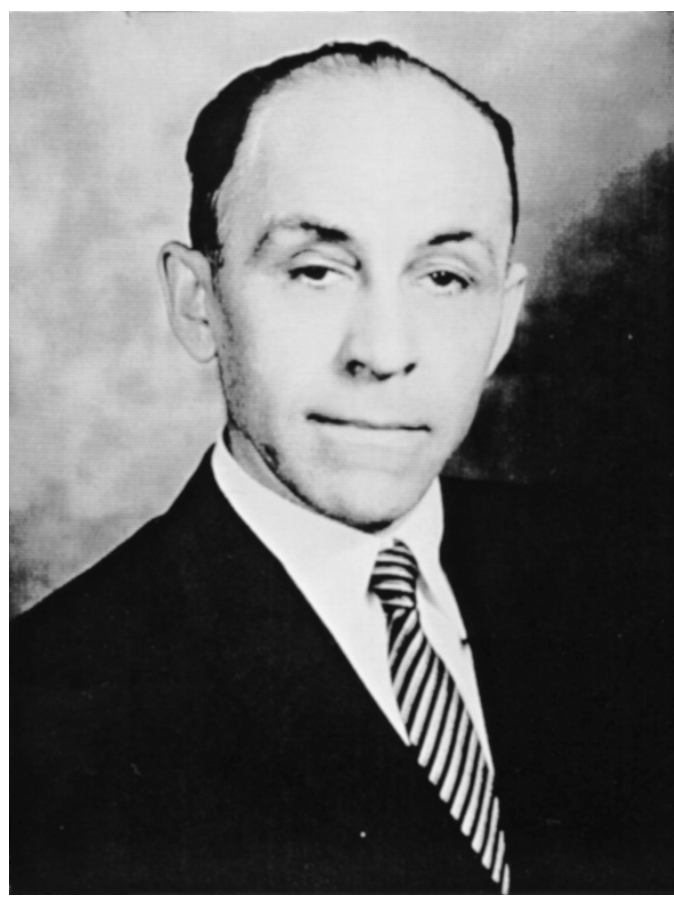

Figure 4. D.W. Woolley, 1963. Courtesy of the Rockefeller University Archives.

an "antimetabolite" of serotonin, Dr. Woolley proposed a role for serotonin in mental illness (Figure 4).

D. W. Woolley received his undergraduate degree from the University of Alberta and his Ph.D. from the University of Wisconsin, where he worked on isolating and characterizing nicotinamide, a substance involved in pellegra. From that work, he developed his theories of specific substances being related to specific human diseases, and described the use of "antimetabolites" substances which are structural analogues of naturally occurring metabolites and which interfere with the functioning of those metabolites-as a tool to investigate those diseases.

Suffering from severe diabetes, Dr. Woolley was entirely blind by the time he joined the faculty at Rockefeller University in 1939. Yet, his many friends and collaborators speak of his ability to "see" things—apparently because he worked very hard at creating his own visual impressions by taking in as much information as he could through his other senses and listening to the descriptions of others. In spite of his blindness, therefore, it is often said that Dr. Woolley "saw" the structure of serotonin in LSD and realized that LSD could be used as an antimetabolite tool to uncover the role of serotonin in mental illnesses. His studies used contraction of rat uterus to show that the effects of serotonin could be blocked by LSD and mimicked by bufotenine. Dr. Woolley also believed that serotonin played a role in brain development, based on its similarity to the plant growth hormone, auxin. Papers on his hypothesis first appeared in the early 1950's (Woolley and Shaw 1954). Although Dr. Woolley had first sent his work to Lancet for publication, it had been rejected, on the basis that it was not enough to make the suggestion, rather the author had to prove his point by curing mental illness. Dr. Woolley's work and hypotheses were summarized in his 1963 book The Biochemical Bases of Psychoses or the Serotonin Hypothesis about Mental Illness (Woolley 1963).

Dr. Page called Dr. Woolley the "most vocal proponent of serotonin's role in brain ... (who) ... made the best case for the participation of serotonin in mental illness." In recognition of his work, Dr. Woolley was elected member of the National Academy of Sciences and The National Academy of Arts and Sciences. He won the Mead Johnson Award, the American Pharmaceutical Association Award, and the Eli Lilly Award, twice. Sadly, Dr. Woolley died while climbing the Andes Mountains, trying to construct a visual impression of the culture of the Incas. He was 52 and it was only three years after his book was published.

\section{CONCLUSIONS}

I hope I have brought to life some of the unique and remarkable people behind the names so well known, on the first serotonin papers. Each of them contributed their own expertise and ideas to the field of serotonin research which would not be what it is today without each one of them. Also, I may have fulfilled Dr. Page's wishes that scientific discoveries should always be learned from the point of view of the people who made them.

\section{ACKNOWLEDGMENTS}

The author wishes to express gratitude to Fred Lautzenheiser of the Cleveland Clinic Archives and The Rockefeller University Archive Center for searching out and supplying material, and to Drs. David Wong, Philip Seeman, and Francesco Erspamer for supplying personal anecdotes. My deepest gratitude goes to Betty Twarog, Maurice Rapport, and Vittorio Erspamer, not only for their help with this article, but also for the discovery of serotonin.

\section{REFERENCES}

Erspamer V, Vialli M (1937): Ricerche sul secreto delle cellule enterocromaffini. Boll d Soc Med-chir Pavia 51:357-363

Erspamer V, Boretti G (1951): Identification and characterization by paper chromatography of enteramine, octopamine, tyramine, histamine and allied substances in extracts of posterior salivary glands of octopoda and in 
other tissue extracts of vertebrates and invertebrates. Arch Int Pharmacodyn 88:296-332

Erspamer V, Asero B (1952): Identification of enteramine, specific hormone of enterochromaffin cells, as 5hydroxtryptamine. Nature 169:800-801

Erspamer V, Ghiretti F (1951): The action of enteramine on the heart of mollusks. J Physiol 115:470-481

Erspamer V (1948): Active substances in the posterior salivary glands of Octopoda. I. Enteramine-like substance. Acta Pharmacol Toxicol 4:213-223

Erspamer V (1946): Presenza di enteramina o di una sostanza enteraminosimile negli estratti gastroenterici delle Ascidie. Experientia 2:369-371

Page IH (1968): Serotonin. Chicago, IL, Yearbook Medical Publishers, Inc

Rapport MM, Green AA, Page IH (1948a): Partial purification of the vasoconstrictor in beef serum. J Biol Chem 174:735-738
Rapport MM, Green AA, Page IH (1948b): Crystalline Serotonin. Science 108:329-330

Rapport MM (1949): Serum vasoconstrictor (serotonin). V. The presence of creatinine in the complex: A proposed structure of the vasoconstrictor principle. J Biol Chem 180:961-969

Twarog BM, Page IH (1953): Serotonin content of some mammalian tissues and urine and a method for its determination. Am J Physiol 175:157-161

Twarog BM (1954): Responses of a molluscan smooth muscle to acetylcholine and 5-hydroxytryptamine. J Cell Comp Physiol 44:141-163

Woolley DW (1963): The Biochemical Bases of Psychoses or the Serotonin Hypothesis about Mental Illness. New York, NY, John Wiley and Sons, Inc

Woolley DW, Shaw E (1954): A biochemical and pharmacological suggestion about certain mental disorders. Proc Natl Acad Sci USA 40:228-231 